\title{
New Exact Solutions to the KdV-Burgers-Kuramoto Equation with the Exp-Function Method
}

\author{
Jae-Myoung Kim and Changbum Chun \\ Department of Mathematics, Sungkyunkwan University, Suwon 440-746, Republic of Korea \\ Correspondence should be addressed to Changbum Chun, cbchun@skku.edu
}

Received 9 February 2012; Accepted 19 March 2012

Academic Editor: Khalida Inayat Noor

Copyright (C) 2012 J.-M. Kim and C. Chun. This is an open access article distributed under the Creative Commons Attribution License, which permits unrestricted use, distribution, and reproduction in any medium, provided the original work is properly cited.

Based on the characteristics of the truncated Painlevé expansion method and the Exp-function method, new generalized solitary wave solutions are constructed for the KdV-Burgers-Kuramoto equation, which cannot be directly constructed from the Exp-function method. This work highlights the power of the Exp-function method in providing generalized solitary wave solutions of different physical structures.

\section{Introduction}

The investigation of the exact solutions of nonlinear partial differential equations plays an important role in mathematics, physics, and other applied science areas. In recent years, a variety of powerful methods has been proposed and analyzed to construct the explicit exact solutions to nonlinear evolution equations. Among these methods, the Exp-function method proposed by $\mathrm{He}$ and $\mathrm{Wu}[1]$ is particularly notable in its power and applicability in solving nonlinear problems, and it has been successfully applied to many kinds of nonlinear partial differential equations [2-17]. All of these applications verified that the Exp-function method is a straightforward, efficient, and versatile technique for finding generalized solitary, periodic, and rational solutions for nonlinear evolution equations as well as for revealing intriguing characteristics of various inner-wave interactions.

The KdV-Burgers-Kuramoto equation $[18,19]$ is

$$
u_{t}+v u u_{x}+\mu u_{x x x}+\alpha u_{x x}+\gamma u_{x x x x}=0,
$$

where $v, \mu, \alpha$, and $\gamma$ are constants. This equation is an important mathematical model arising in many different physical contexts to describe many phenomena which are simultaneously involved in nonlinearity, dissipation, dispersion, and instability, especially at the description 
of turbulence processes. Since the solutions possess their actual physical application [20], various effective methods have been applied to construct the exact solutions of the KdVBurgers-Kuramoto equation. These methods include the tanh function method [21-23], the homogeneous balance method [24], and the generalized F-expansion method [20]. As a consequence, it is still a significant and interesting task to search for new explicit exact solutions for nonlinear evolution equations. In the present work we extend the Exp-function method in combination with the truncated Painlevé expansion method $[25,26]$ to obtain new nontrivial exact solitary wave solutions to the KdV-Burgers-Kuramoto equation (1.1). As a result, we have found some new exact solitary wave solutions to (1.1) in the case where the Exp-function method provides trivial solutions only, which will be investigated in more detail in the following section.

\section{Solitary Wave Solutions by the Exp-Function Method}

Using the transformation $u=u(\eta), \eta=k x+\omega t$, (1.1) becomes the ordinary differential equation:

$$
\omega u^{\prime}+k v u u^{\prime}+k^{2} \alpha u^{\prime \prime}+k^{3} \mu u^{\prime \prime \prime}+k^{4} \gamma u^{\prime \prime \prime \prime}=0 .
$$

According to the Exp-function method [1], we assume that the solution of (2.1) can be expressed in the form

$$
u(\eta)=\frac{\sum_{n=-d}^{c} a_{n} e^{n \eta}}{\sum_{m=-q}^{p} b_{m} e^{m \eta}}=\frac{a_{-d} e^{-d \eta}+\cdots+a_{c} e^{c \eta}}{b_{-q} e^{-q \eta}+\cdots+b_{p} e^{p \eta}}
$$

where $c, d, p$, and $q$ are positive integers, $a_{n}$ and $b_{m}$ are unknown constants, which are to be determined later.

By balancing the linear term of the highest order in (2.1) with the highest order nonlinear term, we get $c+3 p=2(p+c)$, and, thus, $p=c$. Similarly, balancing the linear term of the lowest order in (2.1) with the lowest order nonlinear term yields $q=d$. Here, the values of $c$ and $d$ can be freely chosen [1]. With the help of Maple, we have obtained only trivial solutions of (1.1) for the cases where (i) $p=c=1$ and $d=q=1$, (ii) $p=c=1$ and $d=q=2$, and (iii) $p=$ $c=2$ and $d=q=1$, but one new solitary wave solution in the case where $p=c=2$ and $d=q=2$, which is given by

$$
u(x, t)=\frac{a_{2} e^{2 \eta}+a_{2} b_{1} e^{\eta}+a_{2} b_{0}+a_{2} b_{-1} e^{-\eta}}{e^{2 \eta}+b_{1} e^{\eta}+b_{0}+b_{-1} e^{-\eta}}
$$

where $\eta=k x+\omega t, k$ and $\omega$ are arbitrary constants, and the coefficients $a_{2}, b_{-1}, b_{0}, b_{1}$ are free parameters. We expect that the other cases for the values of $c$ and $d$ will produce nontrivial solitary wave solutions to (1.1). In the next section we will consider finding new exact solitary wave solutions to (1.1) in the case where the Exp-function method provides trivial solutions only, particularly when $p=c=1$ and $q=d=1$. 


\section{New Solitary Wave Solutions to the KdV-Burgers-Kuramoto Equation}

Let us consider the KdV-Burgers-Kuramoto equation defined by (1.1). The truncated Painlevé expansion method will be adopted to find a dependent variable transformation for finding solitary wave solutions to the considered equation. Substituting $u(x, t)$ in the form

$$
u(x, t)=\frac{u_{0}(x, t)}{f(x, t)^{r}}+\frac{u_{1}(x, t)}{f(x, t)^{r-1}}+\cdots+u_{r}(x, t)
$$

into (1.1) and finding the order of the pole $r=2$ for the solution $u(x, t)$ and the functions $u_{0}(x, t), u_{1}(x, t), \ldots, u_{r}(x, t)$, we obtain the dependent variable transformation

$$
u(x, t)=\frac{60 \gamma}{v} \frac{\partial^{3} \ln f}{\partial x^{3}}+\frac{15 \alpha}{v} \frac{\partial^{2} \ln f}{\partial x^{2}}+\left(\frac{240 \mu}{76 v}-\frac{15 \alpha^{2}}{76 v \gamma}\right) \frac{\partial \ln f}{\partial x}
$$

where $\gamma \nu \neq 0$. Throughout the remainder of this paper we assume that $\gamma$ and $\mu$ satisfy the condition $\gamma \mathcal{v} \neq 0$.

Substitution of this transformation into the KdV-Burgers-Kuramoto equation (1.1) yields a rather complicated system of nonlinear equations in $f$ for which we seek the solution form of the Exp-function method [1]:

$$
f(x, t)=\frac{\sum_{n=-c}^{d} a_{n} e^{n \eta}}{\sum_{m=-p}^{q} b_{m} e^{m \eta}},
$$

where $\eta=k x+\omega t$.

For the sake of simplicity, we choose $p=c=1$ and $q=d=1$. Based on the consideration in (3.2) and (3.3), in this paper we assume that (1.1) admits a generalized solitary wave solution of the form

$$
u(x, t)=\frac{g(x, t)}{76 v \gamma\left(b_{1} e^{\eta}+b_{0}+b_{-1} e^{-\eta}\right)^{3}\left(a_{1} e^{\eta}+a_{0}+a_{-1} e^{-\eta}\right)^{3}},
$$

where

$$
\begin{aligned}
g(x, t)= & A_{5} e^{5 \eta}+A_{4} e^{4 \eta}+A_{3} e^{3 \eta}+A_{2} e^{2 \eta}+A_{1} e^{\eta}+A_{0} \\
& +B_{1} e^{-\eta}+B_{2} e^{-2 \eta}+B_{3} e^{-3 \eta}+B_{4} e^{-4 \eta}+B_{5} e^{-5 \eta}
\end{aligned}
$$


where

$$
\begin{aligned}
& A_{0}=-15\left(-12 \alpha^{2} a_{-1}^{2} b_{1}^{2} b_{0} a_{0}-6 \alpha^{2} a_{-1} b_{1}^{2} b_{-1} a_{0}^{2}+6384 \gamma^{2} k^{2} a_{1}^{2} b_{0}^{2} a_{-1} b_{-1}-6 \alpha^{2} a_{-1} b_{1} b_{0}^{2} a_{0}^{2}\right. \\
& +14592 \gamma^{2} k^{2} a_{1}^{2} b_{-1}^{2} a_{-1} b_{1}-684 \gamma k a_{1}^{2} b_{0}^{2} \alpha b_{-1} a_{-1}+1824 \gamma^{2} k^{2} a_{1}^{2} b_{0} b_{-1}^{2} a_{0} \\
& -6384 \gamma^{2} k^{2} a_{1} b_{0}^{2} a_{-1}^{2} b_{1}-684 \gamma k a_{1} b_{0}^{2} \alpha a_{-1}^{2} b_{1}-2 \alpha^{2} a_{-1}^{3} b_{1}^{3}+6384 \gamma^{2} k^{2} a_{1} b_{-1}^{2} a_{0}^{2} b_{1} \\
& +684 \gamma k a_{0}^{2} b_{1} \alpha b_{-1}^{2} a_{1}+684 \gamma k a_{0}^{2} b_{-1} \alpha b_{1}^{2} a_{-1}+456 \gamma k a_{0}^{3} b_{1} \alpha b_{-1} b_{0} \\
& -1824 \gamma^{2} k^{2} a_{-1}^{2} b_{1}^{2} b_{0} a_{0}-456 \gamma k a_{1} b_{0}^{3} \alpha a_{-1} a_{0}+192 \mu \gamma a_{-1}^{2} b_{1}^{2} b_{0} a_{0}-6 \alpha^{2} a_{-1}^{2} b_{1}^{2} b_{-1} a_{1} \\
& +12 \alpha^{2} a_{1}^{2} b_{0} b_{-1}^{2} a_{0}+6 \alpha^{2} a_{0}^{2} b_{1} b_{-1}^{2} a_{1}-1824 \gamma^{2} k^{2} a_{0}^{2} b_{-1} b_{0}^{2} a_{1}+96 \mu \gamma a_{-1} b_{1} b_{0}^{2} a_{0}^{2} \\
& -6384 \gamma^{2} k^{2} a_{-1} b_{1}^{2} a_{0}^{2} b_{-1}-96 \mu \gamma a_{-1} b_{0}^{2} b_{-1} a_{1}^{2}-32 \mu \gamma a_{1}^{3} b_{-1}^{3}-96 \mu \gamma a_{1}^{2} b_{1} b_{-1}^{2} a_{-1} \\
& -192 \mu \gamma a_{1}^{2} b_{0} b_{-1}^{2} a_{0}+96 \mu \gamma a_{-1}^{2} b_{1}^{2} b_{-1} a_{1}+96 \mu \gamma a_{-1}^{2} b_{1} b_{0}^{2} a_{1} \\
& +1824 \gamma^{2} k^{2} a_{-1} b_{1} b_{0}^{2} a_{0}^{2}+32 \mu \gamma a_{-1}^{3} b_{1}^{3}-96 \mu \gamma a_{1} b_{1} b_{-1}^{2} a_{0}^{2}-96 \mu \gamma a_{1} b_{0}^{2} b_{-1} a_{0}^{2} \\
& +96 \mu \gamma a_{-1} b_{1}^{2} b_{-1} a_{0}^{2}-14592 \gamma^{2} k^{2} a_{1} b_{-1} a_{-1}^{2} b_{1}^{2}+2 \alpha^{2} a_{1}^{3} b_{-1}^{3}+6 \alpha^{2} a_{1}^{2} b_{1} b_{-1}^{2} a_{-1} \\
& \left.+6 \alpha^{2} a_{-1} b_{0}^{2} b_{-1} a_{1}^{2}-6 \alpha^{2} a_{-1}^{2} b_{1} b_{0}^{2} a_{1}+6 \alpha^{2} a_{0}^{2} b_{-1} b_{0}^{2} a_{1}\right) k, \\
& A_{1}=-15\left(9 \alpha^{2} a_{-1} b_{0} b_{-1}^{2} a_{1}^{2}+9 \alpha^{2} a_{0}^{2} b_{-1}^{2} b_{0} a_{1}+96 \mu \gamma a_{-1}^{2} b_{1} b_{0} b_{-1} a_{1}+6 \alpha^{2} a_{-1} b_{1} b_{-1}^{2} a_{1} a_{0}\right. \\
& -9 \alpha^{2} a_{-1}^{2} b_{1} b_{0}^{2} a_{0}+\alpha^{2} a_{0}^{3} b_{1} b_{-1}^{2}-6 \alpha^{2} a_{-1}^{2} b_{1} b_{0} b_{-1} a_{1}-9 \alpha^{2} a_{-1}^{2} b_{1}^{2} b_{-1} a_{0}+\alpha^{2} a_{0}^{3} b_{-1} b_{0}^{2} \\
& -5 \alpha^{2} a_{-1}^{3} b_{1}^{2} b_{0}+6 \alpha^{2} a_{-1} b_{0}^{2} b_{-1} a_{1} a_{0}-\alpha^{2} a_{-1}^{2} b_{0}^{3} a_{1}+5 \alpha^{2} a_{0} b_{-1}^{3} a_{1}^{2}-6 \alpha^{2} a_{-1} b_{1} b_{0} b_{-1} a_{0}^{2} \\
& -\alpha^{2} a_{-1} b_{0}^{3} a_{0}^{2}+80 \mu \gamma a_{-1}^{3} b_{1}^{2} b_{0}+304 \gamma k a_{0}^{3} b_{1} \alpha b_{-1}^{2}-456 \gamma k a_{1} b_{0} \alpha a_{-1}^{2} b_{1} b_{-1} \\
& -1824 \gamma^{2} k^{2} a_{1} b_{0}^{2} a_{-1} a_{0} b_{-1}+76 \alpha \gamma k a_{0}^{3} b_{-1} b_{0}^{2}+456 \gamma k a_{1} b_{-1}^{2} \alpha a_{-1} b_{1} a_{0}+304 \gamma^{2} k^{2} a_{-1} b_{0}^{3} a_{0}^{2} \\
& -304 \gamma^{2} k^{2} a_{0}^{3} b_{-1} b_{0}^{2}+16 \mu \gamma a_{-1} b_{0}^{3} a_{0}^{2}-16 \mu \gamma a_{0}^{3} b_{-1} b_{0}^{2}-76 \alpha \gamma k a_{-1} b_{0}^{3} a_{0}^{2} \\
& +96 \mu \gamma a_{-1} b_{1} b_{0} b_{-1} a_{0}^{2}-16 \mu \gamma a_{0}^{3} b_{1} b_{-1}^{2}-912 \gamma k a_{1} b_{0}^{2} \alpha a_{-1} b_{-1} a_{0}-304 \gamma^{2} k^{2} a_{-1}^{3} b_{1}^{2} b_{0} \\
& -14592 \gamma^{2} k^{2} a_{1} b_{-1} a_{-1}^{2} b_{1} b_{0}+8208 \gamma^{2} k^{2} a_{1}^{2} b_{-1}^{2} a_{-1} b_{0}+14592 \gamma^{2} k^{2} a_{1} b_{-1}^{2} a_{-1} b_{1} a_{0} \\
& -96 \mu \gamma a_{-1} b_{1} b_{-1}^{2} a_{1} a_{0}+1824 \gamma^{2} k^{2} a_{-1} b_{0} a_{0}^{2} b_{1} b_{-1}+2432 \gamma^{2} k^{2} a_{0}^{3} b_{1} b_{-1}^{2} \\
& +144 \mu \gamma a_{-1}^{2} b_{1}^{2} b_{-1} a_{0}+684 \gamma k a_{-1}^{2} b_{1}^{2} \alpha b_{-1} a_{0}-144 \mu \gamma a_{1} b_{0} b_{-1}^{2} a_{0}^{2}+144 \mu \gamma a_{-1}^{2} b_{1} b_{0}^{2} a_{0} \\
& -80 \mu \gamma a_{1}^{2} b_{-1}^{3} a_{0}-96 \mu \gamma a_{-1} b_{0}^{2} b_{-1} a_{1} a_{0}+16 \mu \gamma a_{-1}^{2} b_{0}^{3} a_{1}-144 \mu \gamma a_{-1} b_{0} b_{-1}^{2} a_{1}^{2} \\
& +912 \gamma k a_{-1} b_{1} \alpha a_{0}^{2} b_{-1} b_{0}+76 \gamma k a_{-1}^{3} b_{1}^{2} \alpha b_{0}-8208 \gamma^{2} k^{2} a_{-1}^{2} b_{1}^{2} a_{0} b_{-1}-304 \gamma k a_{1} b_{0}^{3} \alpha a_{-1}^{2} \\
& \left.-76 \gamma k a_{1}^{2} b_{-1}^{3} \alpha a_{0}+304 \gamma^{2} k^{2} a_{1}^{2} b_{-1}^{3} a_{0}-684 \gamma k a_{1}^{2} b_{0} \alpha b_{-1}^{2} a_{-1}-2432 \gamma^{2} k^{2} a_{1} b_{0}^{3} a_{-1}^{2}\right) k, \\
& A_{2}=-15\left(-12 \alpha^{2} a_{-1}^{2} b_{1} b_{0} b_{-1} a_{0}-4 \alpha^{2} a_{-1}^{3} b_{1} b_{0}^{2}-4 \alpha^{2} a_{-1}^{3} b_{1}^{2} b_{-1}+12 \alpha^{2} a_{-1} b_{0} b_{-1}^{2} a_{1} a_{0}\right. \\
& -2 \alpha^{2} a_{-1}^{2} b_{0}^{3} a_{0}+2 \alpha^{2} a_{0}^{3} b_{-1}^{2} b_{0}+4 \alpha^{2} a_{1}^{2} b_{-1}^{3} a_{-1}+912 \alpha \gamma k a_{-1}^{2} b_{1} b_{0} b_{-1} a_{0}+4 \alpha^{2} a_{0}^{2} b_{-1}^{3} a_{1}
\end{aligned}
$$




$$
\begin{aligned}
& -304 \gamma^{2} k^{2} a_{-1}^{2} b_{0}^{3} a_{0}+304 \gamma^{2} k^{2} a_{-1}^{3} b_{1} b_{0}^{2}+2432 \gamma^{2} k^{2} a_{1}^{2} b_{-1}^{3} a_{-1}-76 \gamma k a_{0}^{2} b_{-1}^{3} \alpha a_{1} \\
& +76 \gamma k a_{-1}^{3} b_{1} \alpha b_{0}^{2}-304 \gamma k a_{1}^{2} b_{-1}^{3} \alpha a_{-1}-684 \gamma k a_{1} b_{0}^{2} \alpha a_{-1}^{2} b_{-1}+8208 \gamma^{2} k^{2} a_{-1} b_{1} a_{0}^{2} b_{-1}^{2} \\
& -32 \mu \gamma a_{0}^{3} b_{-1}^{2} b_{0}-304 \gamma^{2} k^{2} a_{1} b_{-1}^{3} a_{0}^{2}+64 \mu \gamma a_{-1}^{3} b_{1} b_{0}^{2}-1824 \gamma^{2} k^{2} a_{-1}^{2} b_{1} b_{0} a_{0} b_{-1} \\
& -76 \gamma k a_{-1}^{2} b_{0}^{3} \alpha a_{0}-912 \alpha \gamma k a_{-1} b_{0} b_{-1}^{2} a_{1} a_{0}-64 \mu \gamma a_{1} b_{-1}^{3} a_{0}^{2}+32 \mu \gamma a_{-1}^{2} b_{0}^{3} a_{0} \\
& +304 \gamma k a_{-1}^{3} b_{1}^{2} \alpha b_{-1}+304 \gamma^{2} k^{2} a_{0}^{3} b_{-1}^{2} b_{0}+76 \gamma k a_{0}^{3} b_{-1}^{2} \alpha b_{0}+64 \mu \gamma a_{-1}^{3} b_{1}^{2} b_{-1} \\
& -64 \mu \gamma a_{1}^{2} b_{-1}^{3} a_{-1}-192 \mu \gamma a_{-1} b_{0} b_{-1}^{2} a_{1} a_{0}+192 \mu \gamma a_{-1}^{2} b_{1} b_{0} b_{-1} a_{0}-2432 \gamma^{2} k^{2} a_{-1}^{3} b_{1}^{2} b_{-1} \\
& \left.+1824 \gamma^{2} k^{2} a_{1} b_{-1}^{2} a_{-1} b_{0} a_{0}+684 \gamma k a_{-1} b_{1} \alpha a_{0}^{2} b_{-1}^{2}-8208 \gamma^{2} k^{2} a_{1} b_{-1} a_{-1}^{2} b_{0}^{2}\right) k ， \\
& A_{3}=-15\left(\alpha^{2} a_{0}^{3} b_{-1}^{3}-\alpha^{2} a_{-1}^{3} b_{0}^{3}-456 \gamma k a_{1} b_{-1}^{3} \alpha a_{0} a_{-1}+3 \alpha^{2} a_{-1}^{2} b_{0} b_{-1}^{2} a_{1}\right. \\
& +48 \mu \gamma a_{-1}^{2} b_{1} b_{-1}^{2} a_{0}-96 \mu \gamma a_{1} b_{-1}^{3} a_{0} a_{-1}+16 \mu \gamma a_{-1}^{3} b_{0}^{3}-48 \mu \gamma a_{-1} b_{0} b_{-1}^{2} a_{0}^{2} \\
& +96 \mu \gamma a_{-1}^{3} b_{1} b_{0} b_{-1}+6 \alpha^{2} a_{0} b_{-1}^{3} a_{1} a_{-1}+1824 \gamma^{2} k^{2} a_{-1} b_{0} a_{0}^{2} b_{-1}^{2} \\
& -1824 \gamma^{2} k^{2} a_{-1}^{2} b_{0}^{2} a_{0} b_{-1}-48 \mu \gamma a_{-1}^{2} b_{0} b_{-1}^{2} a_{1}-3 \alpha^{2} a_{-1}^{2} b_{0}^{2} b_{-1} a_{0}-16 \mu \gamma a_{0}^{3} b_{-1}^{3} \\
& -684 \gamma k a_{1} b_{-1}^{2} \alpha a_{-1}^{2} b_{0}+684 \gamma k a_{-1}^{2} b_{1} \alpha a_{0} b_{-1}^{2}+48 \mu \gamma a_{-1}^{2} b_{0}^{2} b_{-1} a_{0} \\
& +6384 \gamma^{2} k^{2} a_{-1}^{2} b_{1} a_{0} \mathrm{~b}_{-1}^{2}-6384 \gamma^{2} k^{2} a_{1} b_{-1}^{2} a_{-1}^{2} b_{0}+456 \gamma k a_{-1}^{3} b_{1} \alpha b_{0} b_{-1} \\
& \left.+3 \alpha^{2} a_{-1} b_{0} b_{-1}^{2} a_{0}^{2}-6 \alpha^{2} a_{-1}^{3} b_{1} b_{0} b_{-1}-3 \alpha^{2} a_{-1}^{2} b_{1} b_{-1}^{2} a_{0}\right) k, \\
& A_{4}=-15\left(-2432 \gamma^{2} k^{2} a_{1} b_{-1}^{3} a_{-1}^{2}-32 \mu \gamma a_{1} b_{-1}^{3} a_{-1}^{2}+2432 \gamma^{2} k^{2} a_{-1}^{3} b_{1} b_{-1}^{2}\right. \\
& +32 \mu \gamma a_{-1}^{3} b_{1} b_{-1}^{2}+304 \alpha \gamma k a_{-1}^{3} b_{1} b_{-1}^{2}-2 \alpha^{2} a_{-1}^{3} b_{0}^{2} b_{-1}+2 \alpha^{2} a_{0}^{2} b_{-1}^{3} a_{-1} \\
& -304 \gamma^{2} k^{2} a_{-1}^{3} b_{0}^{2} b_{-1}+76 \gamma k a_{-1}^{3} b_{0}^{2} \alpha b_{-1}+304 \gamma^{2} k^{2} a_{0}^{2} b_{-1}^{3} a_{-1}-76 \gamma k a_{0}^{2} b_{-1}^{3} \alpha a_{-1} \\
& +2 \alpha^{2} a_{1} b_{-1}^{3} a_{-1}^{2}-32 \mu \gamma a_{0}^{2} b_{-1}^{3} a_{-1}-2 \alpha^{2} a_{-1}^{3} b_{1} b_{-1}^{2}+32 \mu \gamma a_{-1}^{3} b_{0}^{2} b_{-1} \\
& \left.-304 \alpha \gamma k a_{1} b_{-1}^{3} a_{-1}^{2}\right) k \text {, } \\
& A_{5}=-15\left(76 \alpha \gamma k a_{-1}^{3} b_{0} b_{-1}^{2}-76 \alpha \gamma k a_{0} b_{-1}^{3} a_{-1}^{2}+\alpha^{2} a_{0} b_{-1}^{3} a_{-1}^{2}+16 \mu \gamma a_{-1}^{3} b_{0} b_{-1}^{2}\right. \\
& \left.+304 \gamma^{2} k^{2} a_{-1}^{3} b_{0} b_{-1}^{2}-304 \gamma^{2} k^{2} a_{0} b_{-1}^{3} a_{-1}^{2}-16 \mu \gamma a_{0} b_{-1}^{3} a_{-1}^{2}-\alpha^{2} a_{-1}^{3} b_{0} b_{-1}^{2}\right) k ， \\
& B_{1}=-15\left(-96 \mu \gamma a_{1}^{2} b_{1} b_{0} b_{-1} a_{-1}-16 \mu \gamma a_{1}^{2} b_{0}^{3} a_{-1}+304 \gamma^{2} k^{2} a_{0}^{3} b_{1} b_{0}^{2}+9 \alpha^{2} a_{1}^{2} b_{1} b_{-1}^{2} a_{0}\right. \\
& -9 \alpha^{2} a_{-1} b_{1}^{2} b_{0} a_{0}^{2}+684 \gamma k a_{1}^{2} b_{-1}^{2} \alpha b_{1} a_{0}+6 \alpha^{2} a_{1}^{2} b_{1} b_{0} b_{-1} a_{-1}-9 \alpha^{2} a_{-1}^{2} b_{1}^{2} b_{0} a_{1} \\
& -\alpha^{2} a_{0}^{3} b_{1} b_{0}^{2}+8208 \gamma^{2} k^{2} a_{1}^{2} b_{-1}^{2} a_{0} b_{1}-304 \gamma^{2} k^{2} a_{1} b_{0}^{3} a_{0}^{2}-80 \mu \gamma a_{1}^{3} b_{0} b_{-1}^{2} \\
& +\alpha^{2} a_{1}^{2} b_{0}^{3} a_{-1}+\alpha^{2} a_{1} b_{0}^{3} a_{0}^{2}+6 \alpha^{2} a_{1} b_{1} b_{0} b_{-1} a_{0}^{2}+9 \alpha^{2} a_{1}^{2} b_{0}^{2} b_{-1} a_{0}-6 \alpha^{2} a_{-1} b_{1} b_{0}^{2} a_{1} a_{0} \\
& -5 \alpha^{2} a_{0} b_{1}^{3} a_{-1}^{2}-\alpha^{2} a_{0}^{3} b_{1}^{2} b_{-1}-6 \alpha^{2} a_{-1} b_{1}^{2} b_{-1} a_{1} a_{0}+5 \alpha^{2} a_{1}^{3} b_{0} b_{-1}^{2} \\
& -912 \gamma k a_{1} b_{0}^{2} \alpha a_{-1} b_{1} a_{0}+144 \mu \gamma a_{-1}^{2} b_{1}^{2} b_{0} a_{1}+304 \gamma k a_{0}^{3} b_{-1} \alpha b_{1}^{2}-144 \mu \gamma a_{1}^{2} b_{1} b_{-1}^{2} a_{0}
\end{aligned}
$$




$$
\begin{aligned}
& -76 \alpha \gamma k a_{1} b_{0}^{3} a_{0}^{2}-16 \mu \gamma a_{1} b_{0}^{3} a_{0}^{2}+16 \mu \gamma a_{0}^{3} b_{1} b_{0}^{2}+2432 \gamma^{2} k^{2} a_{1}^{2} b_{0}^{3} a_{-1}+76 \alpha \gamma k a_{0}^{3} b_{1} b_{0}^{2} \\
& -304 \gamma^{2} k^{2} a_{-1}^{2} b_{1}^{3} a_{0}+96 \mu \gamma a_{-1} b_{1}^{2} b_{-1} a_{1} a_{0}-96 \mu \gamma a_{1} b_{1} b_{0} b_{-1} a_{0}^{2}+80 \mu \gamma a_{-1}^{2} b_{1}^{3} a_{0} \\
& +144 \mu \gamma a_{-1} b_{1}^{2} b_{0} a_{0}^{2}+912 \gamma k a_{1} b_{-1} \alpha a_{0}^{2} b_{1} b_{0}-76 \gamma k a_{-1}^{2} b_{1}^{3} \alpha a_{0}+456 \gamma k a_{1} b_{-1} \alpha a_{0} b_{1}^{2} a_{-1} \\
& +1824 \gamma^{2} k^{2} a_{1} b_{0}^{2} a_{-1} a_{0} b_{1}-1824 \gamma^{2} k^{2} a_{1} b_{0} a_{0}^{2} b_{1} b_{-1}+76 \gamma k a_{1}^{3} b_{0} \alpha b_{-1}^{2}-304 \gamma k a_{1}^{2} b_{0}^{3} \alpha a_{-1} \\
& -456 \gamma k a_{1}^{2} b_{0} \alpha b_{1} b_{-1} a_{-1}-684 \gamma k a_{1} b_{0} \alpha a_{-1}^{2} b_{1}^{2}+14592 \gamma^{2} k^{2} a_{1}^{2} b_{0} b_{-1} a_{-1} b_{1} \\
& -14592 \gamma^{2} k^{2} a_{1} b_{-1} a_{-1} b_{1}^{2} a_{0}-144 \mu \gamma a_{1}^{2} b_{0}^{2} b_{-1} a_{0}+96 \mu \gamma a_{-1} b_{1} b_{0}^{2} a_{1} a_{0}+16 \mu \gamma a_{0}^{3} b_{1}^{2} b_{-1} \\
& \left.-2432 \gamma^{2} k^{2} a_{0}^{3} b_{1}^{2} b_{-1}+304 \gamma^{2} k^{2} a_{1}^{3} b_{0} b_{-1}^{2}-8208 \gamma^{2} k^{2} a_{1} b_{0} a_{-1}^{2} b_{1}^{2}\right) k, \\
& B_{2}=-15\left(-4 \alpha^{2} a_{0}^{2} b_{1}^{3} a_{-1}-2 \alpha^{2} a_{0}^{3} b_{1}^{2} b_{0}-4 \alpha^{2} a_{-1}^{2} b_{1}^{3} a_{1}+4 \alpha^{2} a_{1}^{3} b_{1} b_{-1}^{2}+4 \alpha^{2} a_{1}^{3} b_{0}^{2} b_{-1}\right. \\
& +1824 \gamma^{2} k^{2} a_{1}^{2} b_{0} b_{-1} a_{0} b_{1}+64 \mu \gamma a_{-1} b_{1}^{3} a_{0}^{2}-8208 \gamma^{2} k^{2} a_{1} b_{-1} a_{0}^{2} b_{1}^{2}+12 \alpha^{2} a_{1}^{2} b_{1} b_{0} b_{-1} a_{0} \\
& +2 \alpha^{2} a_{1}^{2} b_{0}^{3} a_{0}-12 \alpha^{2} a_{-1} b_{1}^{2} b_{0} a_{1} a_{0}-912 \alpha \gamma k a_{-1} b_{1}^{2} b_{0} a_{1} a_{0}-304 \gamma^{2} k^{2} a_{1}^{3} b_{0}^{2} b_{-1} \\
& -76 \gamma k a_{0}^{2} b_{1}^{3} \alpha a_{-1}-1824 \gamma^{2} k^{2} a_{1} b_{0} a_{-1} b_{1}^{2} a_{0}+304 \gamma^{2} k^{2} a_{1}^{2} b_{0}^{3} a_{0}+8208 \gamma^{2} k^{2} a_{1}^{2} b_{0}^{2} a_{-1} b_{1} \\
& +304 \gamma^{2} k^{2} a_{-1} b_{1}^{3} a_{0}^{2}+304 \gamma k a_{1}^{3} b_{-1}^{2} \alpha b_{1}+76 \gamma k a_{1}^{3} b_{0}^{2} \alpha b_{-1}-192 \mu \gamma a_{1}^{2} b_{1} b_{0} b_{-1} a_{0} \\
& +912 \alpha \gamma k a_{1}^{2} b_{1} b_{0} b_{-1} a_{0}-76 \gamma k a_{1}^{2} b_{0}^{3} \alpha a_{0}+192 \mu \gamma a_{-1} b_{1}^{2} b_{0} a_{1} a_{0}-304 \gamma k a_{-1}^{2} b_{1}^{3} \alpha a_{1} \\
& +64 \mu \gamma a_{-1}^{2} b_{1}^{3} a_{1}-32 \mu \gamma a_{1}^{2} b_{0}^{3} a_{0}-64 \mu \gamma a_{1}^{3} b_{0}^{2} b_{-1}-64 \mu \gamma a_{1}^{3} b_{1} b_{-1}^{2}-304 \gamma^{2} k^{2} a_{0}^{3} b_{1}^{2} b_{0} \\
& +76 \gamma k a_{0}^{3} b_{1}^{2} \alpha b_{0}-2432 \gamma^{2} k^{2} a_{-1}^{2} b_{1}^{3} a_{1}+684 \gamma k a_{1} b_{-1} \alpha a_{0}^{2} b_{1}^{2}+2432 \gamma^{2} k^{2} a_{1}^{3} b_{-1}^{2} b_{1} \\
& \left.-684 \gamma k a_{1}^{2} b_{0}^{2} \alpha b_{1} a_{-1}+32 \mu \gamma a_{0}^{3} b_{1}^{2} b_{0}\right) k, \\
& B_{3}=-15\left(\alpha^{2} a_{1}^{3} b_{0}^{3}-\alpha^{2} a_{0}^{3} b_{1}^{3}+3 \alpha^{2} a_{1}^{2} b_{1} b_{0}^{2} a_{0}-3 \alpha^{2} a_{0}^{2} b_{1}^{2} b_{0} a_{1}+3 \alpha^{2} a_{1}^{2} b_{1}^{2} b_{-1} a_{0}-48 \mu \gamma a_{1}^{2} b_{1} b_{0}^{2} a_{0}\right. \\
& +96 \mu \gamma a_{-1} b_{1}^{3} a_{1} a_{0}-16 \mu \gamma a_{1}^{3} b_{0}^{3}-48 \mu \gamma a_{1}^{2} b_{1}^{2} b_{-1} a_{0}-3 \alpha^{2} a_{1}^{2} b_{1}^{2} b_{0} a_{-1}+6 \alpha^{2} a_{1}^{3} b_{1} b_{0} b_{-1} \\
& -96 \mu \gamma a_{1}^{3} b_{1} b_{0} b_{-1}-6384 \gamma^{2} k^{2} a_{1}^{2} b_{-1} a_{0} b_{1}^{2}-456 \gamma k a_{-1} b_{1}^{3} \alpha a_{0} a_{1}-6 \alpha^{2} a_{0} b_{1}^{3} a_{1} a_{-1} \\
& -1824 \gamma^{2} k^{2} a_{1} b_{0} a_{0}^{2} b_{1}^{2}+456 \gamma k a_{1}^{3} b_{0} \alpha b_{1} b_{-1}+1824 \gamma^{2} k^{2} a_{1}^{2} b_{0}^{2} a_{0} b_{1}-684 \gamma k a_{1}^{2} b_{0} \alpha a_{-1} b_{1}^{2} \\
& +684 \gamma k a_{1}^{2} b_{-1} \alpha a_{0} b_{1}^{2}+48 \mu \gamma a_{1}^{2} b_{1}^{2} b_{0} a_{-1}+6384 \gamma^{2} k^{2} a_{1}^{2} b_{0} a_{-1} b_{1}^{2}+48 \mu \gamma a_{1} b_{1}^{2} b_{0} a_{0}^{2} \\
& \left.+16 \mu \gamma a_{0}^{3} b_{1}^{3}\right) k, \\
& B_{4}=-15\left(-304 \alpha \gamma k a_{-1} b_{1}^{3} a_{1}^{2}+304 \alpha \gamma k a_{1}^{3} b_{1}^{2} b_{-1}-32 \mu \gamma a_{1}^{3} b_{1}^{2} b_{-1}+32 \mu \gamma a_{0}^{2} b_{1}^{3} a_{1}\right. \\
& -304 \gamma^{2} k^{2} a_{0}^{2} b_{1}^{3} a_{1}+2432 \gamma^{2} k^{2} a_{-1} b_{1}^{3} a_{1}^{2}-2432 \gamma^{2} k^{2} a_{1}^{3} b_{1}^{2} b_{-1}-32 \mu \gamma a_{1}^{3} b_{1} b_{0}^{2} \\
& +32 \mu \gamma a_{-1} b_{1}^{3} a_{1}^{2}+76 \gamma k a_{1}^{3} b_{0}^{2} \alpha b_{1}-76 \gamma k a_{0}^{2} b_{1}^{3} \alpha a_{1}-2 \alpha^{2} a_{0}^{2} b_{1}^{3} a_{1}-2 \alpha^{2} a_{-1} b_{1}^{3} a_{1}^{2} \\
& \left.+304 \gamma^{2} k^{2} a_{1}^{3} b_{0}^{2} b_{1}+2 \alpha^{2} a_{1}^{3} b_{1}^{2} b_{-1}+2 \alpha^{2} a_{1}^{3} b_{1} b_{0}^{2}\right) k,
\end{aligned}
$$




$$
\begin{gathered}
B_{5}=-15\left(-16 \mu \gamma a_{1}^{3} b_{1}^{2} b_{0}-304 \gamma^{2} k^{2} a_{1}^{3} b_{1}^{2} b_{0}+304 \gamma^{2} k^{2} a_{0} b_{1}^{3} a_{1}^{2}-\alpha^{2} a_{0} b_{1}^{3} a_{1}^{2}+76 \alpha \gamma k a_{1}^{3} b_{1}^{2} b_{0}\right. \\
\left.-76 \alpha \gamma k a_{0} b_{1}^{3} a_{1}^{2}+\alpha^{2} a_{1}^{3} b_{1}^{2} b_{0}+16 \mu \gamma a_{0} b_{1}^{3} a_{1}^{2}\right) k .
\end{gathered}
$$

Substituting (3.4) into (1.1) and equating the coefficients of all powers of $e^{n \eta}$ to zero yields a system of algebraic equations for $a_{1}, a_{0}, a_{-1}, b_{1}, b_{0}, b_{-1}, k$ and $\omega$. Solving the system with the help of Maple, we obtain the following cases.

Case 1. Consider

$$
\left\{a_{1}=0, a_{0}=\frac{a_{-1} b_{1}}{b_{0}}, a_{-1}=a_{-1}, b_{1}=b_{1}, b_{0}=b_{0}, b_{-1}=0, k=k, \omega=\omega\right\} .
$$

Case 2. Consider

$$
\begin{aligned}
& \left\{a_{1}=\frac{a_{0} b_{0}}{b_{-1}}, a_{0}=a_{0}, a_{-1}=0, b_{1}=0, b_{0}=b_{0}\right. \\
& \left.b_{-1}=b_{-1}, k=k, \omega=\frac{k^{2}\left(15 \alpha^{2}-76 \alpha k \gamma-76 \gamma^{2} k^{2}-316 \mu \gamma\right)}{76 \gamma}\right\} .
\end{aligned}
$$

Case 3. Consider

$$
\begin{aligned}
& \left\{a_{1}=\frac{a_{0} b_{0}}{b_{-1}}, a_{0}=a_{0}, a_{-1}=0, b_{1}=0, b_{0}=b_{0}\right. \\
& \left.b_{-1}=b_{-1}, k= \pm \frac{\sqrt{-\mu \gamma}}{\gamma}, \omega=\frac{\mu\left(240 \mu \gamma \pm 76 \alpha \sqrt{-\mu \gamma}-15 \alpha^{2}\right)}{76 \gamma^{2}}\right\} .
\end{aligned}
$$

Case 4. Consider

$$
\begin{aligned}
& \left\{a_{1}=\frac{a_{0} b_{0}}{b_{-1}}, a_{0}=a_{0}, a_{-1}=0, b_{1}=0, b_{0}=b_{0},\right. \\
& b_{-1}=b_{-1}, k=\frac{1}{4} \frac{-19 \alpha \gamma \pm \sqrt{437 \alpha^{2} \gamma^{2}-1216 \gamma^{3} \mu}}{38 \gamma^{2}}, \\
& \omega=-\frac{20848 \mu \alpha^{2} \gamma+39936 \mu^{2} \gamma^{2}+707 \alpha^{4}}{184832 \gamma^{3}} \\
& \left.-\frac{1168 \gamma \alpha \mu \sqrt{19} \sqrt{23 \alpha^{2}-64 \mu \gamma}-41 \alpha^{3} \sqrt{19} \sqrt{23 \alpha^{2}-64 \mu \gamma}}{184832 \gamma^{3}}\right\} .
\end{aligned}
$$


Substituting these cases into (3.4) we obtain the following new solitary wave solutions to (1.1):

$$
\begin{aligned}
u(x, t) & =\frac{15 k}{76 \gamma v} \frac{c_{3} e^{6 \eta}+c_{2} e^{5 \eta}+c_{1} e^{4 \eta}+c_{0} e^{3 \eta}+c_{-1} e^{2 \eta}+c_{-2} e^{\eta}+c_{-3}}{\left(b_{0}+b_{1} e^{\eta}\right)^{6}}, \\
c_{-3} & =\left(\alpha^{2}-16 \mu \gamma\right) b_{1}^{6}, \quad c_{-2}=\left(6 \alpha^{2}-96 \mu \gamma\right) b_{1}^{5} b_{0}, \quad c_{-1}=\left(15 \alpha^{2}-240 \mu \gamma\right) b_{0}^{2} b_{1}^{4}, \\
c_{0} & =\left(20 \alpha^{2}-320 \mu \gamma\right) b_{0}^{3} b_{1}^{3}, \quad c_{1}=\left(15 \alpha^{2}-240 \mu \gamma\right) b_{1}^{2} b_{0}^{4}, \\
c_{2} & =\left(6 \alpha^{2}-96 \mu \gamma\right) b_{0}^{5} b_{1}, \quad c_{3}=\left(\alpha^{2}-16 \mu \gamma\right) b_{0}^{6},
\end{aligned}
$$

where $\eta=k x+\omega t, k$ and $\omega$ are determined in Case $1, b_{0}$ and $b_{1}$ are arbitrary constants. Consider

$$
\begin{aligned}
u(x, t) & =-\frac{15 k}{76 \gamma v} \frac{c_{3} e^{6 \eta}+c_{2} e^{5 \eta}+c_{1} e^{4 \eta}+c_{0} e^{3 \eta}+c_{-1} e^{2 \eta}+c_{-2} e^{\eta}+c_{-3}}{\left(b_{0} e^{\eta}+b_{-1}\right)^{6}}, \\
c_{-3} & =\left(\alpha^{2}-16 \mu \gamma\right) b_{0}^{6}, \quad c_{-2}=\left(6 \alpha^{2}-96 \mu \gamma\right) b_{0}^{5} b_{-1}, \quad c_{-1}=\left(15 \alpha^{2}-240 \mu \gamma\right) b_{-1}^{2} b_{0}^{4}, \\
c_{0} & =\left(20 \alpha^{2}-320 \mu \gamma\right) b_{0}^{3} b_{-1}^{3}, \quad c_{1}=\left(15 \alpha^{2}-240 \mu \gamma\right) b_{-1}^{4} b_{0}^{2}, \\
c_{2} & =\left(6 \alpha^{2}-96 \mu \gamma\right) b_{0} b_{-1}^{5}, \quad c_{3}=\left(\alpha^{2}-16 \mu \gamma\right) b_{-1}^{6},
\end{aligned}
$$

where $\eta=k x+\omega t, k$ and $\omega$ are determined in Case $2, b_{0}$ and $b_{-1}$ are arbitrary constants. Consider

$$
\begin{aligned}
u(x, t) & =-\frac{15 k}{76 \gamma v} \frac{c_{3} e^{6 \eta}+c_{2} e^{5 \eta}+c_{1} e^{4 \eta}+c_{0} e^{3 \eta}+c_{-1} e^{2 \eta}+c_{-2} e^{\eta}+c_{-3}}{\left(b_{0} e^{\eta}+b_{-1}\right)^{6}}, \\
c_{-3} & =\left(\alpha^{2}-16 \gamma \mu\right) b_{0}^{6}, \quad c_{-2}=\left(6 \alpha^{2}-96 \gamma \mu\right) b_{0}^{5} b_{-1}, \quad c_{-1}=\left(15 \alpha^{2}-240 \mu \gamma\right) b_{0}^{4} b_{-1}^{2}, \\
c_{0} & =\left(20 \alpha^{2}-320 \mu \gamma\right) b_{0}^{3} b_{-1}^{3}, \quad c_{1}=\left(15 \alpha^{2}-240 \mu \gamma\right) b_{-1}^{4} b_{0}^{2}, \\
c_{2} & =\left(6 \alpha^{2}-96 \gamma \mu\right) b_{-1}^{5} b_{0}, \quad c_{3}=\left(\alpha^{2}-16 \gamma \mu\right) b_{-1}^{6},
\end{aligned}
$$


where $\eta=k x+\omega t, k$ and $\omega$ are determined in Case $3, b_{0}$ and $b_{-1}$ are arbitrary constants. Consider

$$
\begin{aligned}
u(x, t) & =-\frac{15 k}{2888 \gamma v} \frac{c_{3} e^{6 \eta}+c_{2} e^{5 \eta}+c_{1} e^{4 \eta}+c_{0} e^{3 \eta}+c_{-1} e^{2 \eta}+c_{-2} e^{\eta}+c_{-3}}{\left(b_{0} e^{\eta}+b_{-1}\right)^{6}}, \\
c_{-3} & =\left(\alpha^{2}-16 \mu \gamma\right) b_{0}^{6}, \quad c_{-2}=\left(6 \alpha^{2}-96 \mu \gamma\right) b_{0}^{5} b_{-1}, \quad c_{-1}=\left(15 \alpha^{2}-240 \mu \gamma\right) b_{0}^{4} b_{-1}^{2}, \\
c_{0} & =\left(20 \alpha^{2}-320 \mu \gamma\right) b_{0}^{3} b_{-1}^{3}, \quad c_{1}=\left(15 \alpha^{2}-240 \mu \gamma\right) b_{0}^{2} b_{-1}^{4}, \\
c_{2} & =\left(6 \alpha^{2}-96 \mu \gamma\right) b_{0} b_{-1}^{5}, \quad c_{3}=\left(\alpha^{2}-16 \mu \gamma\right) b_{-1}^{6},
\end{aligned}
$$

where $\eta=k x+\omega t, k$ and $\omega$ are determined in Case $4, b_{0}$ and $b_{-1}$ are arbitrary constants.

The correctness of solutions (3.11)-(3.14) is ensured by testing them on computer with the aid of symbolic computation software Maple. It should be noted that these solutions have not been found in the literature. We also applied the Exp-function method to the KdVBurgers-Kuramoto equation (1.1) to obtain its solitary wave solutions. As a result, we found only trivial solutions for the cases (i) $p=c=q=d=1$, (ii) $p=c=2, q=d=1$, (iii) $p=c=1$, $q=d=2$, and one nontrivial solution for the case $p=c=2, q=d=2$, and we expect that more nontrivial solutions will be found for other cases. This indicates that the solutions obtained in this work cannot be directly constructed by the Exp-function method particularly in the case where $p=c=q=d=1$.

\section{Conclusion}

In this paper, we have successfully implemented the Exp-function method based on the truncated Painlevé expansion method and obtained new generalized solitary wave solutions of the KdV-Burgers-Kuramoto equation. Our approach yields new travelling wave solutions with some free parameters. The approach considered in this paper and the Exp-function method complement each other in that our approach may provide nontrivial solutions in the cases that the Exp-function method may provide only trivial solutions. The result reveals that the Exp-function method is a promising tool because it can provide a variety of new soliton solutions with distinct physical structures.

\section{Acknowledgment}

This research was supported by Basic Science Research Program through the National Research Foundation of Korea (NRF) funded by the Ministry of Education, Science and Technology (2011-0025877).

\section{References}

[1] J.-H. He and X.-H. Wu, "Exp-function method for nonlinear wave equations," Chaos, Solitons and Fractals, vol. 30, no. 3, pp. 700-708, 2006. 
[2] S. D. Zhu, "Exp-function method for the Hybrid-Lattice system," International Journal of Nonlinear Sciences and Numerical Simulation, vol. 8, no. 3, pp. 461-464, 2007.

[3] S. D. Zhu, "Exp-function method for the discrete mKdV lattice," International Journal of Nonlinear Sciences and Numerical Simulation, vol. 8, no. 3, pp. 465-468, 2007.

[4] S. Zhang, "Explicit and exact nontravelling wave solutions of Konopelchenko-Dubrovsky equations," Zeitschrift fur Naturforschung A, vol. 62, no. 12, pp. 689-697, 2007.

[5] S. Zhang, "Application of Exp-function method to a KdV equation with variable coefficients," Physics Letters A, vol. 365, no. 5-6, pp. 448-453, 2007.

[6] S. Zhang, "Exp-function method: solitary, periodic and rational wave solutions of nonlinear evolution equations," Nonlinear Science Letters A, vol. 1, no. 2, pp. 143-146, 2010.

[7] A. Bekir and A. Boz, "Exact solutions for a class of nonlinear partial differential equations using expfunction method," International Journal of Nonlinear Sciences and Numerical Simulation, vol. 8, no. 4, pp. 505-512, 2007.

[8] J. H. He, "An elementary introduction to recently developed asymptotic methods and nanomechanics in textile engineering," International Journal of Modern Physics B, vol. 22, no. 21, pp. 3487-3578, 2008.

[9] X. W. Zhou, Y. X. Wen, and J. H. He, "Exp-function method to solve the nonlinear dispersive $K(m, n)$ equations," International Journal of Nonlinear Sciences and Numerical Simulation, vol. 9, no. 3, pp. 301306, 2008.

[10] C. Q. Dai and Y. Y. Wang, "Exact travelling wave solutions of toda lattice equations obtained via the exp-function method," Zeitschrift fur Naturforschung A, vol. 63, no. 10-11, pp. 657-662, 2008.

[11] A. Bekir and A. Boz, "Exact solutions for nonlinear evolution equations using Exp-function method," Physics Letters A, vol. 372, no. 10, pp. 1619-1625, 2008.

[12] C. Chun, "Solitons and periodic solutions for the fifth-order KdV equation with the Exp-function method," Physics Letters A, vol. 372, no. 16, pp. 2760-2766, 2008.

[13] J.-H. He and L.-N. Zhang, "Generalized solitary solution and compacton-like solution of the JaulentMiodek equations using the Exp-function method," Physics Letters A, vol. 372, no. 7, pp. 1044-1047, 2008.

[14] A. Ebaid, "Exact solitary wave solutions for some nonlinear evolution equations via Exp-function method," Physics Letters A, vol. 365, no. 3, pp. 213-219, 2007.

[15] M. A. Noor, K. I. Noor, A. Waheed, and E. A. Al-Said, "Some new solitonary solutions of the modified Benjamin-Bona-Mahony equation," Computers E Mathematics with Applications, vol. 62, no. 4, pp. 21262131, 2011.

[16] E. M. E. Zayed and M. A. M. Abdelaziz, "Exact solutions for the nonlinear Schrödinger equation with variable coefficients using the generalized extended tanh-function, the sine-cosine and the exp-function methods," Applied Mathematics and Computation, vol. 218, no. 5, pp. 2259-2268, 2011.

[17] K. Parand and J. A. Rad, "Exp-function method for some nonlinear PDE's and a nonlinear ODE's," Journal of King Saud University, vol. 24, no. 1, pp. 1-10, 2012.

[18] Y. Kuramoto and T. Tsuzuki, "Persistent propagation of concentration waves in dissipative media far from thermal equilibrium," Progress of Theoretical Physics, vol. 55, no. 2, pp. 356-369, 1967.

[19] T. Kawahara and M. Tanaka, "Interactions of traveling fronts: an exact solution of a nonlinear diffusion equation," Physics Letters A, vol. 97, no. 8, pp. 311-314, 1983.

[20] S. Zhang, "New exact solutions of the KdV-Burgers-Kuramoto equation," Physics Letters A, vol. 358, no. 5-6, pp. 414-420, 2006.

[21] E. Yusufoğlu and A. Bekir, "The tanh and the sine-cosine methods for exact solutions of the MBBM and the Vakhnenko equations," Chaos, Solitons \& Fractals, vol. 38, no. 4, pp. 1126-1133, 2008.

[22] E. Fan and H. Zhang, "A note on the homogeneous balance method," Physics Letters A, vol. 246, no. 5, pp. 403-406, 1998.

[23] H. B. Lan and K. L. Wang, "Exact solutions for two nonlinear equations. I," Journal of Physics A, vol. 23, no. 17, pp. 3923-3928, 1990.

[24] L. Yang and K. Q. Yang, "Solitary wave solutions of generalized Kuramoto-Sivashinsky equations," Journal of Lanzhou University, vol. 34, no. 4, pp. 53-55, 1998 (Chinese).

[25] J. Weiss, M. Tabor, and G. Carnevale, "The Painlevé property for partial differential equations," Journal of Mathematical Physics, vol. 24, no. 3, pp. 522-526, 1983.

[26] P. A. Clarkson and E. L. Mansfield, "On a shallow water wave equation," Nonlinearity, vol. 7, no. 3, pp. 975-1000, 1994. 


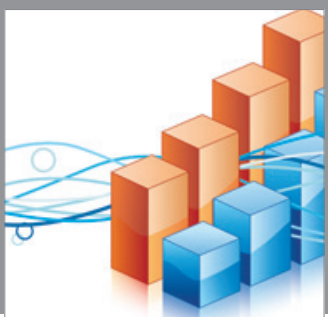

Advances in

Operations Research

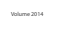

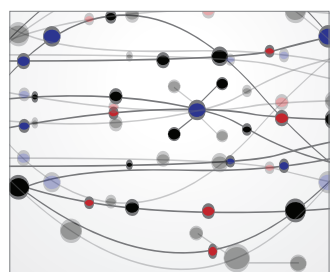

\section{The Scientific} World Journal
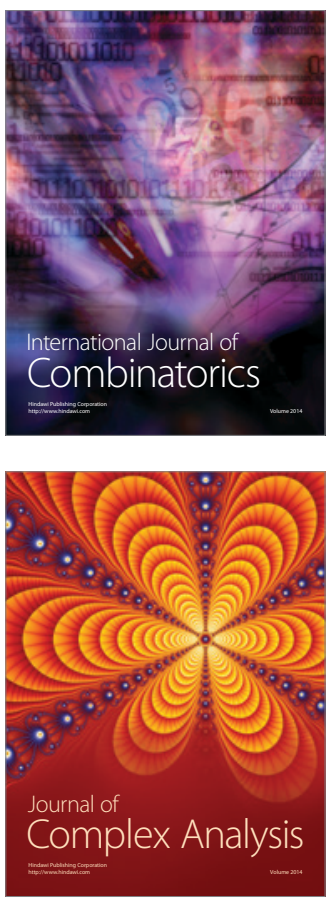

International Journal of

Mathematics and

Mathematical

Sciences
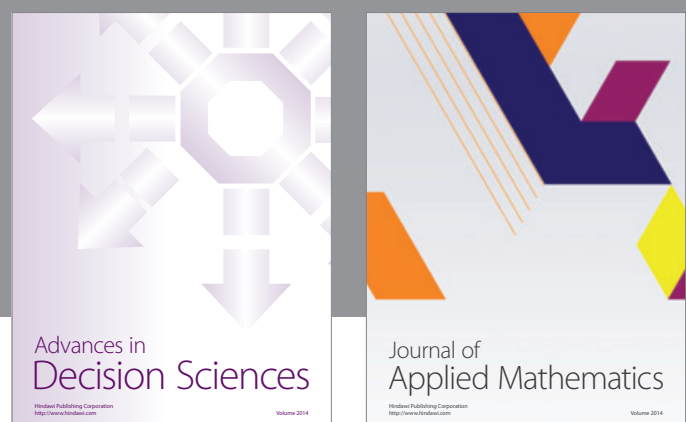

Journal of

Applied Mathematics
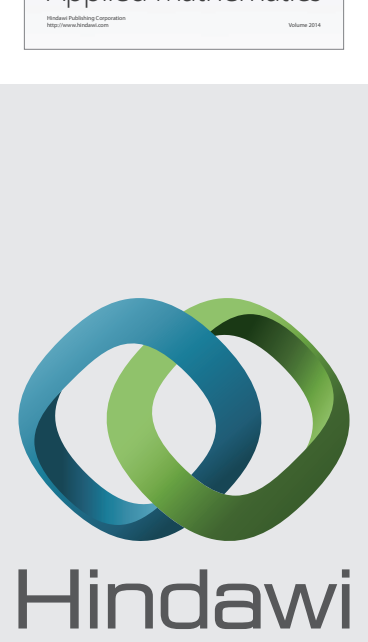

Submit your manuscripts at http://www.hindawi.com
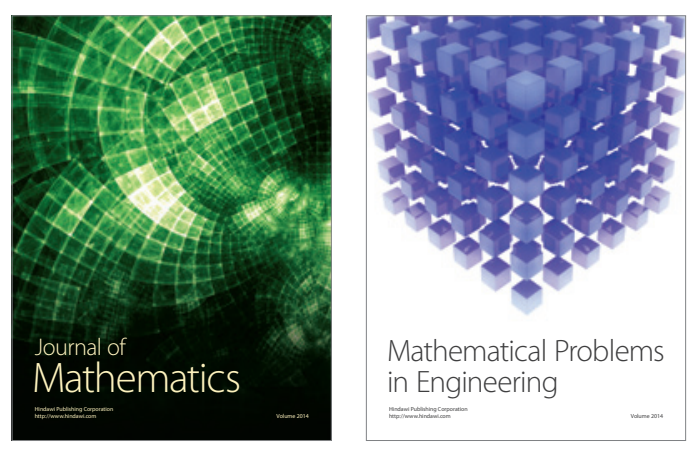

Mathematical Problems in Engineering
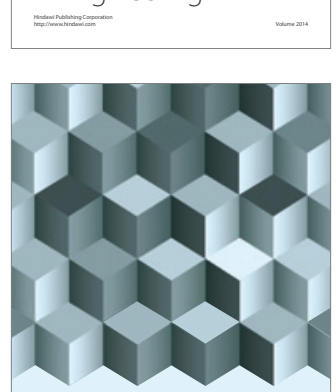

Journal of

Function Spaces
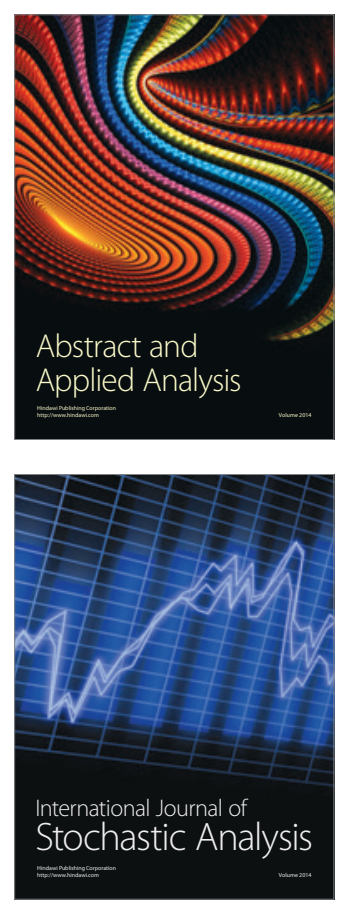

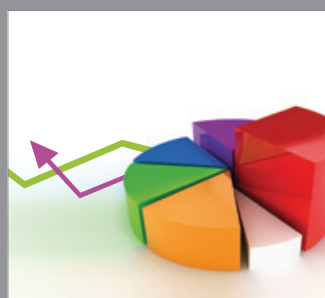

ournal of

Probability and Statistics

Promensencen
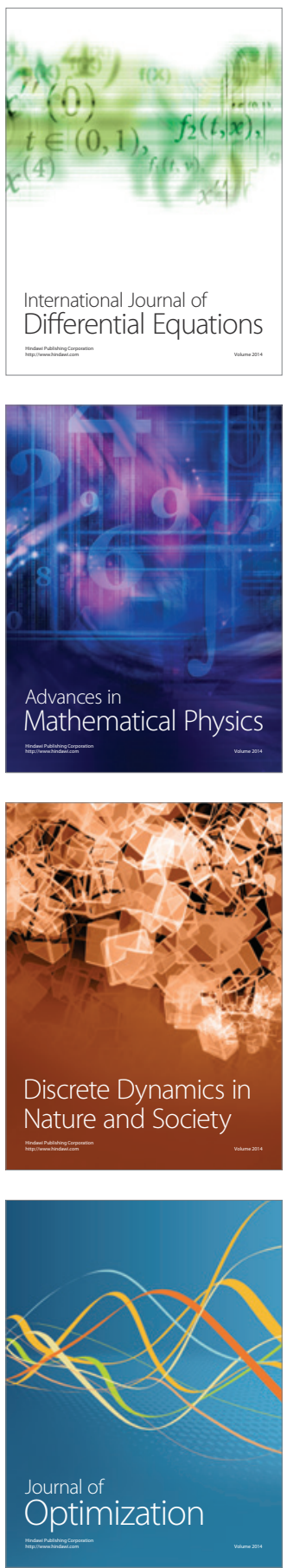